\title{
Antropometria de idosos residentes no município de Joinville-SC, Brasil
}

Anthropometry of elderly living in the city of J oinville-SC, Brazil

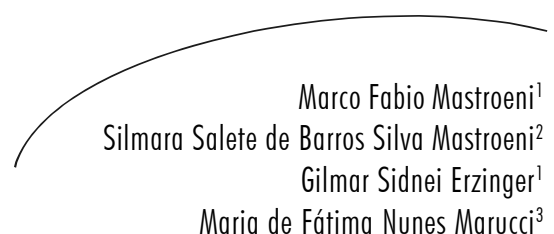

Resumo

Objetivo: Fornecer dados antropométricos de idosos residentes na área urbana do município de Joinville, SC. Metodologia: Foram analisados 218 idosos com idade igual ou superior a 60 anos, de ambos os sexos, não institucionalizados e residentes na área urbana da cidade de Joinville-SC, no ano de 2002. Os idosos foram apresentados segundo sexo e grupo etário: 60-69 anos, 70-79 anos e 80 e mais. As variáveis antropométricas foram apresentadas sob a forma de média, desvio-padrão e percentil (P5, P10, P25, P50, P75, P90 e P95). Resultados: Dos 218 idosos, $130(59,6 \%)$ foram mulheres e $88(40,4 \%)$ homens. Apesar de os valores médios da maioria das variáveis investigadas sugerirem uma tendência a diminuir com o aumento da idade, não houve diferença estatisticamente significativa dos valores médios entre os grupos etários para ambos os sexos $(p>0,05)$. Quando comparados por sexo, os valores médios de IMC, CQ e DCT das mulheres foram significativamente superiores aos dos homens. Estes, em contrapartida, apresentaram valor médio da CC significativamente superior ao das mulheres $(\mathrm{p}<0,05)$. Conclusão: A maioria dos estudos realizados com idosos em outros países tem investigado esta categoria considerando pessoas com idade igual ou superior a 65 anos, o que dificulta a comparação com os dados brasileiros. Somado a outros estudos realizados com idosos no Brasil, os dados obtidos neste estudo podem contribuir para a construção de um padrão antropométrico da população idosa brasileira, ainda inexistente no país.

\footnotetext{
Universidade da Região de Joinville. Programa de Mestrado em Saúde e Meio Ambiente. Joinville, SC, Brasil

2 Associação Educacional Luterana Bom Jesus/IELUSC. Curso de Nutrição. Joinville, SC, Brasil

3 Universidade de São Paulo. Faculdade de Saúde Pública, Departamento de Nutrição. São Paulo, SP, Brasil
}

Fonte de financiamento: Fundação de Amparo à Pesquisa da Universidade da Região de JoinvilleUNIVILLE. Processo no 26/02 de 28/02/2002.

Artigo baseado na tese de Doutorado "Estado nutricional e consumo de macronutrientes de idosos da cidade de Joinville, SC. 2002", defendida junto ao Departamento de Nutrição da Faculdade de Saúde Pública da Universidade de São Paulo em fevereiro de 2004. 109 p.

Correspondência / Correspondence

Marco Fabio Mastroeni

Universidade da Região de Joinville, Programa de Mestrado em Saúde e Meio Ambiente. Campus

Universitário S/N, Bairro Bom Retiro - 89223-251 - Joinville, SC, Brasil

E-mail: marco.mastroeni@gmail.com

\section{Palavras-chave:}

Antropometria. Estado nutricional. Idoso.

Joinville, SC 


\section{Abstract}

Objective: To provide anthropometric data of elderly people living in the urban area of Joinville, SC. Methodology: Two hundred and eighteen elderly people, aged 60 years or more, of both sexes, not institutionalized and residents in the urban area of Joinville city-SC were analyzed, in the year of 2002. The elderly were gathered according to sex and age group (60-69 years, 70-79 years and 80 and more). The anthropometric variables are presented under the average form, deviation-pattern and percentile (P5, P10, P25, P50, P75, P90 and P95). Results: From 218 elderly, 130 (59.6\%) were women and 88 (40.4\%) men. Although the mean values of most investigated variables were proposing a tendency to reduce with the age increase, there was no statistically significant difference in the mean values among the age groups for both sex $(p>0.05)$. When compared by sex, the body mass index, hip circumference and skinfolds mean values of women were significantly higher from the men. These, however, presented the waist circumference mean value significantly higher from the women $(p<0.05)$. Conclusion: Most of the studies accomplished with elderly people in other countries have been investigated this category considering people with 65 years old or more, what makes difficult the comparison with the Brazilian data. In addition to other studies with elderly people in Brazil, the data obtained in this study can contribute for the construction of an anthropometric pattern of the Brazilian elderly population, inexistent in the country so far.
Key words:

Anthropometry.

Nutritional Status. Aged. Joinville city

\section{INTRODUÇÃOO}

A antropometria é um método não invasivo que objetiva determinar e monitorar o peso corporal, a composição corporal e avaliar a distribuição de gordura corporal, relacionando-a com o desenvolvimento de doenças crônicas. ${ }^{1}$ Dentre as técnicas de avaliação do estado nutricional, a antropometria tem sido muito utilizada em estudos epidemiológicos, devido principalmente ao seu baixo custo e fácil aplicação. Considerado o método mais utilizado em avaliação nutricional de idosos, os dados antropométricos frequentemente utilizados para este grupo etário compreendem peso, estatura, dobras cutâneas e circunferências do braço, da cintura, do quadril e da panturrilha. ${ }^{2}$

As alterações na composição corporal de um indivíduo ao longo dos anos são um processo normal que ocorre em função da idade, apesar de o grau de alteração variar entre os indivíduos. As principais alterações referem-se à massa magra e aos padrões de distribuição de gordura. ${ }^{2} \mathrm{~A}$ estatura tende a diminuir cerca de $1-2 \mathrm{~cm} /$ década, sendo essa diminuição mais acentuada em idosos.
O peso, além de variar segundo o sexo também diminui com o avanço da idade, e essa redução inclui os declínios da massa muscular e da massa celular em geral. O Índice de Massa Corporal (IMC) também diminui após os 70 anos de idade. ${ }^{3}$

Avaliar o estado nutricional de idosos através de medidas antropométricas requer o uso de valores de referência para comparação. Alguns países já apresentaram estudos definindo valores de referência antropométrica para pessoas acima de 60 anos. Entre esses países, destacam-se os Estados Unidos, a Inglaterra, a França, Itália e o México. ${ }^{4-10}$ No Brasil, existem alguns estudos que investigaram as características antropométricas da população ${ }^{11-17}$ mas ainda não existem valores antropométricos de referência para idosos. Somado às dimensões continentais do país, estudos que descrevam as características antropométricas da população tornam-se imprescindíveis para o Brasil estabelecer seu próprio padrão antropométrico nos diferentes grupos etários.

Este estudo teve como objetivo fornecer dados antropométricos de idosos residentes na área urbana 
do município de Joinville-SC, de forma a contribuir para a construção de um padrão antropométrico de brasileiros com 60 e mais anos de idade.

\section{METODOLOGIA}

Os dados apresentados fazem parte de um estudo maior, ${ }^{18}$ de base populacional, transversal, realizado mediante inquérito domiciliar e que avaliou o estado nutricional e o consumo de macronutrientes de idosos da cidade de JoinvilleSC, mediante avaliações antropométrica, bioquímica e dietética.

\section{Amostra}

Participaram deste estudo idosos com idade igual ou superior a 60 anos, de ambos os sexos, não institucionalizados e residentes na área urbana da cidade de Joinville-SC, no ano de 2002. Foram excluídos do estudo os idosos que apresentaram debilidade clínica grave, os que estavam em fase terminal de doença e os que não foram localizados após o terceiro contato efetuado por telefone e pessoalmente na residência. ${ }^{18}$

\section{Coleta dos dados}

$\mathrm{Na}$ primeira etapa do estudo, que consistiu da coleta de dados em domićlio, foram entrevistados 660 idosos. Nesta fase, foram coletados dados sociodemográficos e econômicos. $\mathrm{Na}$ segunda etapa do estudo, os idosos foram convidados a comparecer aos Postos de Saúde de sua região, no período da manhã, para a coleta dos dados antropométricos, bioquímicos e dietéticos. Nesta etapa, todos os idosos que compareceram aos postos de saúde foram avaliados por profissionais e estudantes da área da saúde previamente treinados.

Os idosos foram agrupados segundo sexo e grupo etário (60-69 anos, 70-79 anos e 80 e mais). As variáveis antropométricas foram apresentadas sob a forma de média, desvio-padrão e percentil (P5, P10, P25, P50, P75, P90 e P95).

\section{Variáveis antropométricas}

As variáveis antropométricas analisadas foram: peso; estatura; IMC; circunferências do braço (CB), da panturrilha (CP), da cintura (CC) e do quadril (CQ); relação cintura-quadril (RCQ) e dobra cutânea tricipital (DCT).

O IMC é um índice que relaciona peso com a estatura, sendo muito utilizado para identificar indivíduos com desnutrição, sobrepeso ou obesidade na população. ${ }^{19}$ As medidas da CB e CP estimam a massa muscular do braço e panturrilha, respectivamente, e têm sido utilizadas como medidas da perda da massa muscular em idosos. Ambas as medidas são consideradas eficientes indicadores de desnutrição para esse grupo etário. ${ }^{3}$ Tanto a medida da CC como a RCQ têm sido utilizadas para diagnosticar risco para doenças cardiovasculares e distúrbios metabólicos..$^{20} \mathrm{ADCT}$ é uma forma indireta de mensuração da gordura corporal, e facilmente aferida. ${ }^{3}$

Para a mensuração do peso, utilizou-se uma balança digital da marca Soehnle ${ }^{\varpi}$, com capacidade de $150 \mathrm{~kg}$ e divisão de $100 \mathrm{~g}$. No momento da mensuração, o idoso estava vestindo o mínimo de roupas possível (com os bolsos vazios, sem casaco, chapéu, boné, cinto, xale e demais acessórios que acarretassem aumento do peso), sem calçados, e posicionado no centro da balança, de forma a distribuir o peso do corpo entre os pés. A estatura foi mensurada utilizando-se estadiômetro portátil da marca Seca ${ }^{\circledR}$ com capacidade para até $200 \mathrm{~cm}$ e precisão de $0,1 \mathrm{~cm}$. O IMC foi calculado a partir das variáveis peso e estatura, onde o peso $(\mathrm{kg})$ foi dividido pela estatura $(\mathrm{m})$ elevada ao quadrado. Ambos, peso e estatura, foram mensurados seguindo-se as técnicas de Frisancho.?

As variáveis $\mathrm{CB}, \mathrm{CC}, \mathrm{CQ}$ e $\mathrm{CP}$ foram mensuradas de acordo com as técnicas de Callaway et al., ${ }^{21}$ utilizando-se fita métrica inextensível da marca Cardiomed ${ }^{\circledast}$, com capacidade para até 150 $\mathrm{cm}$ e precisão de $0,1 \mathrm{~cm}$. Para as medidas da $\mathrm{CB}$, CC e CQ, no momento da mensuração o voluntário foi posicionado em pé, de forma ereta, com os braços soltos ao lado do tronco, as palmas 
das mãos voltadas para o mesmo, e vestindo camisa sem mangas. A medida da CB foi realizada após o avaliador marcar o ponto médio do braço direito da pessoa, posicionado paralelo ao lado do tronco. O ponto médio foi localizado através da flexão do cotovelo, de forma que a palma da mão ficasse voltada para cima, formando um ângulo de $90^{\circ}$ no cotovelo. A circunferência da cintura foi medida com a fita posicionada ao redor da menor curvatura localizada entre as costelas e a crista ilíaca. Para a mensuração da circunferência do quadril, a fita foi posicionada ao redor da região do quadril, na área de maior protuberância. A medida da RCQ foi obtida através da divisão da CC pela CQ.

A mensuração da DCT foi efetuada com o auxílio de um compasso de dobras cutâneas da marca Cescorf ${ }^{\boxplus}$ com variação de 0,1 mm. Para a tomada da medida, o avaliador localizou o ponto médio do braço esquerdo, identificado para a mensuração do perímetro do braço. Em seguida, o avaliador foi posicionado atrás do voluntário e, com os dedos polegar e indicador, pinçou a pele a, aproximadamente, $1 \mathrm{~cm}$ acima do ponto médio, efetuando a medida com o compasso exatamente sobre o ponto médio, seguindo-se as técnicas de Harrison et al. ${ }^{22}$

Todas as mensurações foram realizadas em duplicata, adotando-se a média dos valores de cada uma na análise dos dados.

\section{Análise estatística}

A análise estatística foi realizada utilizando-se o programa Statistical Package for the Social Science (SPSS), versão 14.0. As variáveis antropométricas são apresentadas sob a forma de média, desvio-padrão e percentil, e organizadas segundo sexo e grupo etário. As diferenças de médias entre os sexos foram verificadas utilizandose o teste $t$ Student para variáveis que apresentaram distribuição normal, e o teste Mann-Whitney quando não houve distribuição normal. Para verificar o efeito do grupo etário nas variáveis antropométricas, foram utilizados os testes ANOVA e a comparação de Tukey. Para verificar se houve semelhança entre o grupo de idosos que não participou da segunda etapa do estudo e o grupo que participou, utilizou-se o teste do Quiquadrado. A normalidade foi verificada através do teste Shapiro-Wilk. O nível de confiabilidade adotado foi de $95 \%$.

\section{Aspecto ético}

Quando houve concordância de participação, os voluntários assinaram o Termo de Consentimento Livre e Esclarecido, elaborado de acordo com as normas da Resolução no 196/1996 do Conselho Nacional de Saúde, ${ }^{23}$ sendo fornecida uma cópia a cada participante do estudo. As dúvidas surgidas ao longo do estudo foram esclarecidas pelo pesquisador responsável. A identificação dos participantes foi sigilosa e os dados ficaram armazenados sob responsabilidade do coordenador da pesquisa. Este estudo foi aprovado pelo Comitê de Ética em Pesquisa da Faculdade de Saúde Pública da Universidade de São Paulo - USP (no 341/2000).

\section{RESULTADOS}

Dos 660 idosos que compuseram a primeira etapa do estudo, na qual foram analisadas as características sociodemográficas, ${ }^{18} 218$ compareceram aos postos de saúde para avaliação antropométrica. Destes, 130 $(59,6 \%)$ foram mulheres e $88(40,4 \%)$ homens. As idades mínima e máxima encontradas foram 60 e 87 anos, respectivamente, sendo a média de idade 68,3 $\pm 6,1$ anos. As mulheres apresentaram média de idade de 68,3 $\pm 5,9$ anos, e os homens de 68,2 $\pm 6,5$ anos.

A tabela 1 mostra os valores de média, desviospadrão e $\mathrm{p}$ das variáveis antropométricas investigadas, segundo sexo e grupo etário. Apesar de os valores médios da maioria das variáveis investigadas sugerirem uma tendência a diminuir com o aumento da idade, não houve diferença estatisticamente significativa $(p<0,05)$ dos valores médios entre os grupos etários, para ambos os sexos. Quando comparados por sexo, os valores médios de IMC, CQ e DCT das mulheres foram significativamente superiores aos dos homens. Estes, em contrapartida, apresentaram o valor médio da CC significativamente superior ao das mulheres $(p<0,05)$. 
Tabela 1 - Médias, desvios-padrão (DP) e valores de $p$ das variáveis antropométricas de idosos da área urbana de Joinville-SC, segundo sexo e grupo etário. Joinville, SC, 2002.

\begin{tabular}{|c|c|c|c|c|c|c|c|}
\hline \multirow{2}{*}{$\begin{array}{c}\text { Variáveis/ } \\
\text { Grupo etário (anos) }\end{array}$} & \multicolumn{3}{|c|}{ Homem } & \multicolumn{3}{|c|}{ Mulher } & \multirow{2}{*}{$p$} \\
\hline & $\mathrm{n}$ & Média & $\mathrm{DP}$ & $\mathrm{n}$ & Média & $\mathrm{DP}$ & \\
\hline \multicolumn{8}{|l|}{ Peso $(\mathrm{kg})$} \\
\hline Total & 87 & 70,19 & 11,82 & 130 & 67,20 & 14,32 & 0,092 \\
\hline $60-69$ & 53 & 70,92 & 10,70 & 81 & 68,39 & 14,41 & \\
\hline $70-79$ & 27 & 69,51 & 14,26 & 43 & 65,41 & 13,45 & \\
\hline$\geqslant 80$ & 7 & 67,22 & 10,66 & 6 & 63,87 & 19,61 & \\
\hline \multicolumn{8}{|l|}{ Estatura $(\mathrm{cm})$} \\
\hline Total & 87 & 165,89 & 7,20 & 130 & 154,98 & 6,90 & 0,743 \\
\hline $60-69$ & 53 & 166,04 & 6,99 & 81 & 155,43 & 6,50 & \\
\hline $70-79$ & 27 & 166,28 & 7,90 & 43 & 154,64 & 7,26 & \\
\hline$\geqslant 80 *$ & 7 & 163,23 & 6,34 & 6 & 151,22 & 9,45 & \\
\hline \multicolumn{8}{|l|}{$\operatorname{IMC}\left(\mathrm{kg} / \mathrm{m}^{2}\right)^{*}$} \\
\hline Total & 87 & 25,46 & 3,69 & 130 & 27,83 & 4,83 & 0,023 \\
\hline $60-69$ & 53 & 25,73 & 3,59 & 81 & 28,19 & 5,09 & \\
\hline $70-79$ & 27 & 25,01 & 4,12 & 43 & 27,20 & 4,30 & \\
\hline$\geqslant 80$ & 7 & 25,17 & 2,97 & 6 & 27,53 & 5,19 & \\
\hline \multicolumn{8}{|l|}{$\mathrm{CB}(\mathrm{cm})$} \\
\hline Total & 88 & 29,83 & 3,39 & 130 & 31,07 & 4,08 & 0,086 \\
\hline $60-69$ & 54 & 30,42 & 3,47 & 81 & 31,50 & 4,17 & \\
\hline $70-79$ & 27 & 29,01 & 3,19 & 43 & 30,57 & 3,79 & \\
\hline$\geqslant 80$ & 7 & 28,44 & 2,91 & 6 & 28,90 & 4,44 & \\
\hline \multicolumn{8}{|l|}{$\mathrm{CP}(\mathrm{cm})$} \\
\hline Total & 88 & 37,31 & 4,79 & 130 & 37,07 & 4,71 & 0,592 \\
\hline $60-69$ & 54 & 37,95 & 4,66 & 81 & 34,05 & 4,34 & \\
\hline $70-79$ & 27 & 36,55 & 5,13 & 43 & 37,01 & 5,25 & \\
\hline$\geqslant 80$ & 7 & 35,30 & 4,10 & 6 & 34,05 & 5,34 & \\
\hline \multicolumn{8}{|l|}{$\mathrm{CC}(\mathrm{cm}) *$} \\
\hline Total & 88 & 94,03 & 10,71 & 130 & 93,77 & 13,33 & 0,039 \\
\hline $60-69$ & 54 & 94,13 & 9,74 & 81 & 94,17 & 13,27 & \\
\hline $70-79$ & 27 & 94,18 & 13,04 & 43 & 92,93 & 12,74 & \\
\hline$\geqslant 80$ & 7 & 92,72 & 9,29 & 6 & 94,35 & 19,90 & \\
\hline \multicolumn{8}{|l|}{$\mathrm{CQ}(\mathrm{cm}) *$} \\
\hline Total & 88 & 97,65 & 6,81 & 130 & 102,82 & 11,29 & 0,001 \\
\hline $60-69$ & 54 & 96,02 & 14,84 & 81 & 103,08 & 11,86 & \\
\hline $70-79$ & 27 & 94,11 & 19,83 & 43 & 102,35 & 9,91 & \\
\hline$\geqslant 80$ & 7 & 96,61 & 3,72 & 6 & 102,65 & 14,50 & \\
\hline \multicolumn{8}{|l|}{ RCQ (cm) } \\
\hline Total & 88 & 0,96 & 0,69 & 130 & 0,91 & 0,08 & 0,190 \\
\hline $60-69$ & 54 & 0,96 & 0,06 & 81 & 0,91 & 0,07 & \\
\hline $70-79$ & 27 & 0,96 & 0,07 & 43 & 0,90 & 0,08 & \\
\hline$\geqslant 80$ & 7 & 0,95 & 0,07 & 6 & 0,91 & 0,09 & \\
\hline \multicolumn{8}{|l|}{$\operatorname{DCT}(\mathrm{mm}) *$} \\
\hline Total & 88 & 10,85 & 4,13 & 130 & 21,53 & 6,04 & 0,001 \\
\hline $60-69$ & 54 & 10,65 & 4,06 & 81 & 21,56 & 6,10 & \\
\hline $70-79$ & 27 & 10,64 & 3,77 & 43 & 21,83 & 5,89 & \\
\hline$\geqslant 80$ & 7 & 13,25 & 5,76 & 6 & 19,05 & 6,85 & \\
\hline
\end{tabular}

IMC: Índice de Massa Corporal; CB: Circunferência do braço; CP: Circunferência da panturrilha; CC: Circunferência da cintura; CQ: Circunferência do quadril (CQ); RCQ: Relação cintura-quadril; DCT: Dobra cutânea tricipital.

*Diferença estatisticamente significativa dos valores médios entre os sexos $(p<0,05)$. 
Para verificar se havia semelhança entre o grupo de idosos que não participou da segunda etapa do estudo (439 idosos não examinados) com o grupo que participou (221 idosos examinados), ambos foram analisados quanto às variáveis: sexo, estado civil, grupo etário, cor ou grupo étnico, escolaridade e renda (tabela 2). O teste do Qui-quadrado mostrou não haver diferença estatisticamente significativa $(p<0,05)$ entre os dois grupos em relação às variáveis investigadas (tabela2).

Tabela 2 - Distribuição das freqüências absoluta (n) e relativa (\%) de idosos em duas amostras da área urbana de Joinville-SC, segundo dados socioeconômicos e demográficos. 2002.

\begin{tabular}{|c|c|c|c|c|c|c|c|}
\hline \multirow{2}{*}{$\begin{array}{c}\text { Dados socioeconômicos e } \\
\text { demográficos }\end{array}$} & \multicolumn{2}{|c|}{$\begin{array}{c}\text { Não examinados } \\
\mathrm{n}=439\end{array}$} & \multicolumn{2}{|c|}{$\begin{array}{c}\text { Examinados } \\
\mathrm{n}=221 \\
\end{array}$} & \multicolumn{2}{|c|}{$\begin{array}{c}\text { Total } \\
\mathrm{n}=660\end{array}$} & \multirow[t]{2}{*}{$p$} \\
\hline & $\mathrm{n}$ & $\%$ & $\mathrm{n}$ & $\%$ & $\mathrm{n}$ & $\%$ & \\
\hline Sexo & & & & & & & 0,4737 \\
\hline Masculino & 190 & 43,3 & 90 & 40,7 & 280 & 42,4 & \\
\hline Feminino & 249 & 56,7 & 131 & 59,3 & 380 & 57,6 & \\
\hline Estado civil & & & & & & & 0,8549 \\
\hline Solteiro & 9 & 2,1 & 3 & 1,4 & 12 & 1,8 & \\
\hline Casado/União consensual & 267 & 60,8 & 142 & 64,2 & 409 & 62,0 & \\
\hline Desquitado/Separado & 14 & 3,2 & 7 & 3,2 & 21 & 3,2 & \\
\hline Viúvo & 149 & 33,9 & 69 & 31,2 & 218 & 33,0 & \\
\hline Escolaridade & & & & & & & 0,3784 \\
\hline Não alfabetizado & 39 & 8,9 & 22 & 10,0 & 61 & 9,2 & \\
\hline Alfabetizado & 39 & 8,9 & 13 & 5,9 & 52 & 7,9 & \\
\hline Ensino fundamental incompleto & 323 & 73,6 & 158 & 71,4 & 481 & 72,8 & \\
\hline Ensino fundamental completo & 16 & 3,6 & 15 & 6,8 & 31 & 4,7 & \\
\hline Ensino médio e superior & 22 & 5,0 & 13 & 5,9 & 35 & 5,4 & \\
\hline Renda $(\mathrm{SM}=\mathrm{R} \$ 200,00)$ & & & & & & & 0,6893 \\
\hline Dependente & 142 & 32,3 & 75 & 33,9 & 217 & 32,8 & \\
\hline Até $2 \mathrm{SM}$ & 203 & 46,2 & 98 & 44,4 & 301 & 45,6 & \\
\hline $2-3 \mathrm{SM}$ & 55 & 12,5 & 25 & 11,3 & 80 & 12,1 & \\
\hline 3-5 SM & 26 & 5,9 & 18 & 8,1 & 44 & 6,7 & \\
\hline $5+\mathrm{SM}$ & 13 & 3,1 & 5 & 2,3 & 18 & 2,8 & \\
\hline
\end{tabular}

SM: Salário mínimo. 
As tabelas 3 e 4 mostram os valores médios antropométricas, segundo grupo etário de dos percentis referentes às variáveis homense mulheres, respectivamente.

Tabela 3 - Percentis das variáveis antropométricas de homens idosos da área urbana de Joinville-SC, segundo grupo etário. Joinville, SC, 2002.

\begin{tabular}{|c|c|c|c|c|c|c|c|c|}
\hline \multirow{2}{*}{ Grupo etário } & \multirow{2}{*}{$\mathrm{n}$} & \multicolumn{7}{|c|}{ Percentis } \\
\hline & & 5 & 10 & 25 & 50 & 75 & 90 & 95 \\
\hline \multicolumn{9}{|l|}{ Peso (kg) } \\
\hline Total & 87 & 50,94 & 54,40 & 63,35 & 68,70 & 77,30 & 85,28 & 93,18 \\
\hline $60-69$ & 53 & 52,97 & 57,91 & 63,75 & 70,00 & 76,05 & 84,27 & 93,06 \\
\hline $70-79$ & 27 & 43,64 & 49,69 & 61,55 & 68,00 & 78,70 & 91,96 & 96,77 \\
\hline$\geqslant 80$ & 7 & 50,90 & 50,90 & 62,05 & 64,45 & 77,25 & 83,75 & 83,75 \\
\hline \multicolumn{9}{|l|}{ Estatura $(\mathrm{cm})$} \\
\hline Total & 87 & 153,70 & 155,12 & 161,40 & 167,45 & 171,05 & 174,13 & 176,65 \\
\hline $60-69$ & 53 & 153,46 & 155,73 & 161,07 & 167,05 & 171,02 & 174,61 & 178,92 \\
\hline $70-79$ & 27 & 145,82 & 154,91 & 161,50 & 167,55 & 172,00 & 174,37 & 175,84 \\
\hline$\geqslant 80$ & 7 & 153,50 & 153,50 & 158,50 & 162,60 & 170,00 & 172,05 & 172,05 \\
\hline \multicolumn{9}{|l|}{$\operatorname{IMC}\left(\mathrm{kg} / \mathrm{m}^{2}\right)$} \\
\hline Total & 87 & 19,46 & 20,82 & 23,60 & 25,40 & 27,30 & 29,92 & 32,98 \\
\hline $60-69$ & 53 & 19,84 & 21,98 & 23,60 & 25,40 & 27,30 & 30,42 & 33,57 \\
\hline $70-79$ & 27 & 16,64 & 19,62 & 21,70 & 24,80 & 27,50 & 30,42 & 33,22 \\
\hline$\geqslant 80$ & 7 & 19,30 & 19,30 & 21,10 & 25,70 & 26,30 & 29,00 & 29,00 \\
\hline \multicolumn{9}{|l|}{$\mathrm{CB}(\mathrm{cm})$} \\
\hline Total & 88 & 23,45 & 26,09 & 28,02 & 29,55 & 31,40 & 31,31 & 35,90 \\
\hline $60-69$ & 54 & 24,95 & 26,30 & 28,27 & 30,15 & 32,35 & 35,75 & 37,02 \\
\hline $70-79$ & 27 & 21,30 & 24,80 & 28,00 & 29,10 & 31,00 & 33,28 & 34,18 \\
\hline$\geqslant 80$ & 7 & 22,90 & 22,90 & 26,40 & 29,10 & 30,60 & 31,50 & 31,50 \\
\hline \multicolumn{9}{|l|}{$\mathrm{CP}(\mathrm{cm})$} \\
\hline Total & 88 & 30,83 & 31,97 & 34,67 & 36,45 & 39,07 & 45,88 & 48,52 \\
\hline $60-69$ & 54 & 31,52 & 32,65 & 35,37 & 36,60 & 39,35 & 46,95 & 49,02 \\
\hline $70-79$ & 27 & 28,30 & 30,94 & 33,00 & 36,00 & 39,30 & 46,06 & 48,06 \\
\hline$\geqslant 80$ & 7 & 28,90 & 28,90 & 32,00 & 37,00 & 38,90 & 40,00 & 40,00 \\
\hline \multicolumn{9}{|l|}{$\mathrm{CC}(\mathrm{cm})$} \\
\hline Total & 88 & 74,84 & 81,47 & 88,02 & 83,90 & 100,50 & 108,96 & 114,03 \\
\hline $60-69$ & 54 & 74,70 & 80,48 & 87,20 & 93,10 & 100,00 & 108,00 & 112,10 \\
\hline $70-79$ & 27 & 28,80 & 67,60 & 87,47 & 93,10 & 101,60 & 114,37 & 116,26 \\
\hline$\geqslant 80$ & 7 & 74,30 & 74,30 & 88,80 & 94,30 & 97,90 & 103,50 & 103,50 \\
\hline \multicolumn{9}{|l|}{$\mathrm{CQ}(\mathrm{cm})$} \\
\hline Total & 88 & 87,44 & 90,28 & 93,42 & 96,75 & 101,40 & 107,02 & 110,43 \\
\hline $60-69$ & 54 & 86,32 & 89,50 & 92,80 & 96,80 & 101,90 & 107,08 & 110,86 \\
\hline $70-79$ & 27 & 37,57 & 83,95 & 92,52 & 96,40 & 101,10 & 107,87 & 113,29 \\
\hline$\geqslant 80$ & 7 & 92,70 & 92,70 & 93,30 & 96,10 & 101,00 & 102,30 & 102,30 \\
\hline \multicolumn{9}{|l|}{$\mathrm{RCQ}(\mathrm{cm})$} \\
\hline Total & 88 & 0,82 & 0,87 & 0,92 & 0,96 & 1,01 & 1,05 & 1,08 \\
\hline $60-69$ & 54 & 0,85 & 0,87 & 0,90 & 0,96 & 1,01 & 1,05 & 1,07 \\
\hline $70-79$ & 27 & 0,78 & 0,85 & 0,93 & 0,96 & 1,01 & 1,05 & 1,12 \\
\hline$\geqslant 80$ & 7 & 0,80 & 0,80 & 0,92 & 0,99 & 1,01 & 1,01 & 1,01 \\
\hline \multicolumn{9}{|l|}{$\mathrm{DCT}(\mathrm{mm})$} \\
\hline Total & 88 & 4,41 & 5,59 & 8,12 & 10,05 & 13,25 & 16,84 & 18,86 \\
\hline $60-69$ & 54 & 4,62 & 5,35 & 8,07 & 9,95 & 13,12 & 17,20 & 18,50 \\
\hline $70-79$ & 27 & 3,32 & 5,30 & 8,30 & 9,90 & 13,10 & 16,32 & 17,04 \\
\hline$\geqslant 80$ & 7 & 7,10 & 7,10 & 7,80 & 11,50 & 21,00 & 21,20 & 21,20 \\
\hline
\end{tabular}

IMC: Índice de Massa Corporal; CB: Circunferência do braço; CP: Circunferência da panturrilha; CC: Circunferência da cintura; CQ: Circunferência do quadril (CQ); RCQ: Relação cintura-quadril; DCT: Dobra cutânea tricipital. 
Tabela 4 - Percentis das variáveis antropométricas de mulheres idosas da área urbana de Joinville-SC, segundo grupo etário. Joinville, SC, 2002.

\begin{tabular}{|c|c|c|c|c|c|c|c|c|}
\hline \multirow{2}{*}{ Grupo etário } & \multirow{2}{*}{$\mathrm{n}$} & \multicolumn{7}{|c|}{ Percentis } \\
\hline & & 5 & 10 & 25 & 50 & 75 & 90 & 95 \\
\hline \multicolumn{9}{|l|}{ Peso (kg) } \\
\hline Total & 130 & 47,60 & 51,31 & 56,22 & 65,62 & 74,91 & 86,96 & 97,77 \\
\hline $60-69$ & 81 & 47,68 & 49,72 & 58,42 & 67,40 & 75,67 & 87,62 & 99,70 \\
\hline $70-79$ & 43 & 46,44 & 51,34 & 55,85 & 64,10 & 72,80 & 84,81 & 94,26 \\
\hline$\geqslant 80$ & 6 & 53,95 & 53,95 & 54,43 & 56,57 & 69,26 & 103,80 & 103,80 \\
\hline \multicolumn{9}{|l|}{ Estatura $(\mathrm{cm})$} \\
\hline Total & 130 & 142,33 & 145,56 & 149,86 & 154,92 & 160,00 & 164,04 & 166,54 \\
\hline $60-69$ & 81 & 143,95 & 145,92 & 150,02 & 155,45 & 160,35 & 163,99 & 165,74 \\
\hline $70-79$ & 43 & 142,24 & 145,82 & 149,50 & 153,50 & 159,00 & 165,99 & 170,68 \\
\hline$\geqslant 80$ & 6 & 142,20 & 142,20 & 143,02 & 148,95 & 159,81 & 166,30 & 166,30 \\
\hline \multicolumn{9}{|l|}{$\operatorname{IMC}\left(\mathrm{kg} / \mathrm{m}^{2}\right)$} \\
\hline Total & 130 & 20,45 & 22,50 & 24,20 & 27,35 & 29,82 & 34,87 & 37,63 \\
\hline $60-69$ & 81 & 20,00 & 21,84 & 24,20 & 28,00 & 31,05 & 34,74 & 37,82 \\
\hline $70-79$ & 43 & 20,26 & 22,74 & 25,00 & 26,40 & 28,90 & 34,06 & 37,58 \\
\hline$\geqslant 80$ & 6 & 23,20 & 23,20 & 23,87 & 26,05 & 30,60 & 37,50 & 37,50 \\
\hline \multicolumn{9}{|l|}{$\mathrm{CB}(\mathrm{cm})$} \\
\hline Total & 130 & 24,56 & 26,00 & 28,67 & 30,80 & 33,07 & 36,97 & 38,59 \\
\hline $60-69$ & 81 & 24,79 & 26,80 & 28,75 & 31,00 & 33,40 & 37,80 & 39,97 \\
\hline $70-79$ & 43 & 24,46 & 25,50 & 28,90 & 30,80 & 32,70 & 35,56 & 38,04 \\
\hline$\geqslant 80$ & 6 & 23,50 & 23,50 & 25,67 & 28,40 & 31,67 & 36,70 & 36,70 \\
\hline \multicolumn{9}{|l|}{$\mathrm{CP}(\mathrm{cm})$} \\
\hline Total & 130 & 30,50 & 31,51 & 33,80 & 36,15 & 40,30 & 44,07 & 45,94 \\
\hline $60-69$ & 81 & 30,54 & 31,84 & 34,80 & 36,50 & 40,35 & 43,80 & 45,99 \\
\hline $70-79$ & 43 & 29,82 & 31,26 & 33,20 & 36,00 & 40,30 & 45,12 & 48,32 \\
\hline$\geqslant 80$ & 6 & 28,30 & 28,30 & 30,70 & 33,20 & 36,52 & 44,10 & 44,10 \\
\hline \multicolumn{9}{|l|}{$\mathrm{CC}(\mathrm{cm})$} \\
\hline Total & 130 & 73,55 & 78,73 & 85,80 & 92,80 & 102,12 & 111,10 & 123,90 \\
\hline $60-69$ & 81 & 71,56 & 78,54 & 86,55 & 95,00 & 102,50 & 112,34 & 122,98 \\
\hline $70-79$ & 43 & 74,68 & 79,68 & 85,50 & 90,50 & 102,10 & 108,58 & 121,86 \\
\hline$\geqslant 80$ & 6 & 77,50 & 77,50 & 80,87 & 90,40 & 102,75 & 133,20 & 133,20 \\
\hline \multicolumn{9}{|l|}{$\mathrm{CQ}(\mathrm{cm})$} \\
\hline Total & 130 & 86,37 & 89,87 & 94,65 & 102,50 & 108,07 & 118,36 & 128,81 \\
\hline $60-69$ & 81 & 85,25 & 88,60 & 94,45 & 102,80 & 108,35 & 119,66 & 130,52 \\
\hline $70-79$ & 43 & 85,16 & 90,42 & 95,60 & 102,50 & 107,10 & 116,50 & 123,06 \\
\hline$\geqslant 80$ & 6 & 90,50 & 90,50 & 91,62 & 99,15 & 111,42 & 129,80 & 129,80 \\
\hline \multicolumn{9}{|l|}{$\mathrm{RCQ}(\mathrm{cm})$} \\
\hline Total & 130 & 0,77 & 0,80 & 0,86 & 0,92 & 0,96 & 1,00 & 1,02 \\
\hline $60-69$ & 81 & 0,79 & 0,80 & 0,87 & 0,92 & 0,96 & 0,99 & 1,00 \\
\hline $70-79$ & 43 & 0,74 & 0,80 & 0,85 & 0,92 & 0,97 & 1,01 & 1,03 \\
\hline$\geqslant 80$ & 6 & 0,78 & 0,78 & 0,82 & 0,91 & 1,02 & 1,03 & 1,03 \\
\hline \multicolumn{9}{|l|}{$\operatorname{DCT}(\mathrm{mm})$} \\
\hline Total & 130 & 10,50 & 12,82 & 17,85 & 21,95 & 25,42 & 28,28 & 30,63 \\
\hline $60-69$ & 81 & 10,50 & 12,84 & 17,50 & 22,60 & 25,35 & 28,38 & 31,64 \\
\hline $70-79$ & 43 & 12,04 & 12,84 & 18,60 & 21,90 & 25,50 & 27,86 & 34,56 \\
\hline$\geqslant 80$ & 6 & 9,80 & 9,80 & 14,07 & 17,90 & 25,40 & 29,00 & 29,00 \\
\hline
\end{tabular}

IMC: Índice de Massa Corporal; CB: Circunferência do braço; CP: Circunferência da panturrilha; CC: Circunferência da cintura; CQ: Circunferência do quadril (CQ); RCQ: Relação cintura-quadril; DCT: Dobra cutânea tricipital. 


\section{DISCUSSÃO}

Este estudo descreve a composição corporal de uma amostra representativa de idosos de Joinville, SC. Os resultados obtidos podem ser considerados representativos da população idosa do município.

A principal característica que pode ter limitado a análise dos dados foi a elevada perda amostral na segunda etapa do estudo. Atribuiu-se esta perda a fatores como: 1) ausência no momento do contato (mesmo após dois contatos, por telefone ou na residência); 2) impossibilidade de se deslocar até o posto de saúde devido a compromissos domiciliares (ir ao mercado, cozinhar, cuidar de netos, etc), e problemas de saúde; 3) não achar necessário participar do estudo por possuir plano de saúde; 4) falecimento, entre outros. Cabe lembrar que, mesmo com a elevada perda amostral, a análise estatística revelou não haver diferença significativa entre o grupo que participou e o que não participou da coleta dos dados antropométricos.

Assim como em outros estudos desenvolvidos no Brasil, ${ }^{11,13,15-17,24,25}$ neste estudo também se verificou maior frequência de idosos do sexo feminino. ${ }^{18}$

$\mathrm{Na}$ literatura, a maioria dos estudos observou valores médios de peso e estatura superiores para o sexo masculino..$^{3,8,11,15}$ Neste estudo, não houve diferença estatisticamente significativa $(p>0,05)$ dos valores médios de peso e estatura nos três grupos etários entre os sexos. Em contrapartida, quando avaliados em relação ao IMC, as mulheres apresentaram valores médios significativamente superiores $\left(27,83 \mathrm{~kg} / \mathrm{m}^{2}\right)$ aos dos homens $\left(25,46 \mathrm{~kg} / \mathrm{m}^{2}\right)$. Adotando-se a classificação da Organização Pan-Americana de Saúde (OPAS), ${ }^{26}$ a qual considera baixo peso IMC $\leq 23 \mathrm{~kg} / \mathrm{m}^{2}$, peso normal $23<\mathrm{IMC}<28 \mathrm{~kg} / \mathrm{m}^{2}$, pré-obesidade $28 \leq \mathrm{IMC} \leq 30 \mathrm{~kg} / \mathrm{m}^{2}$ e obesidade $\mathrm{IMC} \geq 30 \mathrm{~kg} / \mathrm{m}^{2}$, homens e mulheres deste estudo foram caracterizados como eutróficos. Vale lembrar que a classificação da OPAS no projeto Saúde, Bemestar e Envelhecimento (SABE) foi construída com base em pesquisas desenvolvidas em países $\mathrm{da}$
América Latina, incluindo o Brasil. Quando utilizada a classificação da Organização Mundial da Saúde (OMS) ${ }^{19}$ para indivíduos com sobrepeso, ainda descrito como IMC igual ou superior a 25,0 $\mathrm{kg} / \mathrm{m}^{2}$, a prevalência deste passou a ser caracterizada para ambos os sexos. Em outros estudos desenvolvidos no Brasil, ${ }^{12,13,16,17,27}$ e em outros países, ${ }^{10,28}$ as mulheres têm mostrado maior prevalência de obesidade, talvez devido aos diferentes pontos de corte adotados, que dificulta a comparação dos dados.

O excesso de peso é um dos principais fatores de risco de uma série de doenças crônicas não transmissíveis, entre elas a hipercolesterolemia, o diabetes, as doenças cardiovasculares, algumas formas de câncer, entre outras, e representa atualmente um preocupante desafio à saúde pública. O sobrepeso é um problema eminentemente urbano, com algumas diferenciações por região. No sul do Brasil, o excesso de peso também é elevado na área rural. Tais resultados podem refletir a presença diferenciada de fatores determinantes de sobrepeso relacionados a condições de vida, história ocupacional e estilo de vida, principalmente atividade física e consumo alimentar, conforme gênero, nas diversas realidades sociais do país. ${ }^{16}$

A CB é utilizada para avaliar a quantidade de gordura subcutânea acumulada no corpo. Alguns estudos revelam que as mulheres apresentam maior quantidade de gordura subcutânea acumulada. ${ }^{27,28}$ Apesar de os valores médios de $\mathrm{CB}$ encontrados neste estudo homens $29,83 \mathrm{~cm}$ e mulheres $31,07 \mathrm{~cm}$ - terem sido similares aos encontrados na pesquisa $\mathrm{SABE}$ em São Paulo $(29,16 \mathrm{~cm}$ e 31,02 cm, respectivamente para homens e mulheres), ${ }^{24}$ para este estudo não houve diferença significativa entre os sexos. Em Fortaleza-CE, Menezes \& Marucci ${ }^{29}$ também não encontraram diferença estatisticamente significativa dos valores médios de CB entre os sexos: 29,3 cm para homens e mulheres, ${ }^{29}$ mostrando que tanto no estudo em Fortaleza como no estudo em Joinville não há diferença da quantidade de gordura subcutânea entre os sexos. 
Assim como a $\mathrm{CB}$, também não houve diferença significativa dos valores médios de $\mathrm{CP}$ entre os sexos: $37,31 \mathrm{~cm}$ para homens e $37,07 \mathrm{~cm}$ para as mulheres. Apesar de os valores médios de CP encontrados neste estudo terem sido superiores, a semelhança dos valores médios entre os sexos também foi verificada nos estudos de Marucci \& Barbosa $^{27} 35,15 \mathrm{~cm}$ para homens e $35,36 \mathrm{~cm}$ para mulheres; e de Rauen et al. ${ }^{17} 31,6$ $\mathrm{cm}$ e $32,0 \mathrm{~cm}$, respectivamente, para homens e mulheres. A CP estima a reserva muscular na perna, e é muito utilizada como indicador de desnutrição e perda de massa muscular em idosos.

Em relação à CC, ainda que a diferença não tenha sido elevada, neste estudo os resultados mostraram valores médios significativamente maiores ( $p>0,05)$ para o sexo masculino: 94,03 $\mathrm{cm}$ e $93,77 \mathrm{~cm}$, respectivamente, para homens e mulheres. Resultado similar foi encontrado no estudo de Marucci \& Barbosa $^{27}$ 95,20 cm para homens e $93,97 \mathrm{~cm}$ para mulheres. Os valores médios de CC podem ser utilizados como indicativos de risco para doenças cardiovasculares e distúrbios metabólicos. A OMS classifica como pontos de corte para CC inadequado os valores e"94,0 cm para homens e e" $80,0 \mathrm{~cm}$ para mulheres. ${ }^{19}$ Analisando-se a CC em percentis, observa-se que, neste estudo, mais do que $75 \%$ das mulheres podem apresentar riscos cardiovasculares, independentemente do grupo etário. Em relação aos homens, esses riscos diminuem para aproximadamente $25 \%$. Santos e Sichieri ${ }^{29}$ encontraram, em média, $39,2 \%$ e $65,9 \%$ para homens e mulheres, respectivamente, em relação à inadequação dos valores médios de CC.

Quanto ao RCQ, adotando-se a classificação da WHO 2000, ${ }^{19}$ a qual estabelece pontos de corte para RCQ adequados valores inferiores a 1,0 para homens, e 0,85 para as mulheres, percebe-se que aproximadamente $25 \%$ dos homens, independentemente do grupo etário, apresentaram risco para o desenvolvimento de doenças cardiovasculares e distúrbios metabólicos. Para as mulheres, excetuando-se o grupo de 80 e mais anos de idade, aproximadamente $90 \%$ apresentaram RCQ igual ou superior a 0,8 - portanto, dentro do grupo de risco.
Apesar de tal resultado apontar para o desenvolvimento de doenças crônicas não transmissíveis, Lean et al. ${ }^{20}$ consideram a variável CC melhor indicador do que a RCQ em relação aos riscos mencionados, e propõe valores iguais ou superiores a 88 e $102 \mathrm{~cm}$ para mulheres e homens, respectivamente.

Ao se observar a variável DCT, também frequentemente utilizada para avaliar o estado nutricional, ${ }^{8}$ neste estudo as mulheres apresentaram significativamente $(\mathrm{p}>0,05)$ maior (21,53 $\mathrm{mm})$ acúmulo de gordura do que os homens $(10,85 \mathrm{~mm})$. Tal resultado foi similar ao encontrado na pesquisa SABE em São Paulo, onde os valores médios de DCT foram 13,71 mm e 25,22 $\mathrm{mm}$, respectivamente, para homens e mulheres. ${ }^{24}$ Menezes \& Marucci ${ }^{29}$ em estudo realizado com idosos institucionalizados no município de Fortaleza-CE, também encontraram valores médios de DCT significativamente ( $>0,05)$ maiores para o sexo feminino: $13,0 \mathrm{~mm}$ e 21,3 mm, respectivamente, para homens e mulheres. O mesmo foi observado por Rauen et al. ${ }^{17}$ em outro estudo realizado no município de FlorianópolisSC com idosos institucionalizados. Similar aos estudos anteriores, esses autores observaram maior $(15,6 \mathrm{~mm})$ acúmulo de gordura nas mulheres em relação aos homens $(10,3 \mathrm{~mm}) .{ }^{17}$ Quando investigados em relação ao percentil 50 , os valores médios de DCT apresentaram uma tendência a diminuir com o aumento da idade, principalmente para as mulheres, corroborando o encontrado em outros estudos. ${ }^{8,11,29-31}$ Para os homens, observou-se diminuição discreta da DCT apenas do grupo etário de 60-69 para o de 70-79 anos, aumentando no grupo de 80 anos e mais de idade.

\section{CONCLUSÃO}

A maioria dos estudos realizados com idosos em outros países tem investigado esta categoria considerando pessoas com idade igual ou superior a 65 anos, o que dificulta a comparação com os dados brasileiros. Há que se considerar, ainda, as variações regionais existentes em um país com dimensões continentais, como é o caso do Brasil. 
Os resultados obtidos neste estudo mostraram elevada prevalência de excesso de peso e risco para o desenvolvimento de doenças cardiovasculares, para ambos os sexos, mas com destaque para as mulheres. Apesar de esta tendência de aumento de peso revelar que a população idosa avaliada está vivenciando o processo de transição nutricional, marcada pela prevalência de excesso de peso, a construção de um padrão antropométrico específico para a esse grupo

\section{REFERÊNCIAS}

1. Kuczmarski MF, Kuczmarski RJ. Nutritional assessment of older adults. In: Schlenker ED. Nutrition in Aging. $3^{\text {rd }}$ ed. Boston: McGrawHill; 1998. p. 261-94.

2. Schlenker ED. Body composition, energy, and physical activity. In: _. Nutrition in Aging. $3^{\text {rd }}$ ed. Boston: McGraw-Hill; 1998. p. 92-119.

3. World Health Organization. Technical Report Series, 854. Physical status: the use and interpretation of anthropometry. Geneva: WHO; 1995. p. 375-409.

4. Burr ML, Phillips KM. Anthropometric norms in the elderly. Br J Nutr 1984; 51: 165-9.

5. Chumlea WC, Roche AF, Mukherjee D. Nutritional assessment of the elderly through anthropometry. Columbus, Ohio: Ross Laboratories; 1987. 47p.

6. Delarue J, Constans T, Malvy D, Pradignac A, Couet C, Lamisse F. Anthropometric values in an elderly French population. Br J Nutr 1994; 71:295-302.

7. Frisancho AR. New standards of weight and body composition by frame size and height for assessment of nutritional status of adults and the elderly. Am J Clin Nutr 1984; 40:808-19.

8. Kuczmarski MF, Kuczmarski RJ, Najjar M. Descriptive anthropometric reference data for older Americans. J Am Diet Assoc 2000; 100(1):59-66.

9. Perissinotto E, Pisent C, Sergi G, Grigoletto F, Enzi G. Anthropometric measurements in the elderly: age and gender differences. Br J Nutr 2002; 87:177-86.

10. Velázquez-Alva MC, Castillo-Martínez L, Irigoyen- Camacho E, Zepeda-Zepeda MA, Gutiérrez- Robledo LM, Cisneros-Moysen P. Estudio antropométrico en un grupo de hombres y mujeres de la tercera edad en la etário no país possibilitará a correta comparação entre os estudos, além de melhorar a qualidade do diagnóstico nutricional de idosos. Também é importante destacar que os resultados observados em Joinville, assim como em outras regiões do Brasil, evidenciam a necessidade de ações mais efetivas no controle desse quadro, de forma a se evitar o aumento do desenvolvimento de doenças crônicas não transmissíveis.
Ciudad de México. Salud Publica Mex 1996; 38:466-74.

11. Barbosa AR, Souza JMP, Lebrão ML, Laurenti R, Marucci MFN. Anthropometry of elderly residents in the city of São Paulo, Brazil. Cad Saude Publica 2005; 21(6):1929-38.

12. Barreto SM, Passos VMA, Costa MFFL. Obesity and underweight among Brazilian elderly. The Bambuí Health and Aging Study. Cad Saude Publica 2003; 19(2):605-12.

13. Cabrera MAS, Jacob Filho W. Obesidade em idosos: prevalência, distribuição e associação com hábitos e co-morbidades. Arq Bras Endocrinol Metabol 2001; 45(5):494-501.

14. Coitinho DC, Leão MM, Recine ESR, Sichieri R. Condições nutricionais da população brasileira: adultos e idosos. Brasília: INAN; 1991. [Pesquisa Nacional sobre Saúde e Nutrição].

15. Menezes TM, Marucci MFN. Antropometria de idosos residentes em instituições geriátricas, Fortaleza, CE. Rev Saude Publica 2005; 39(2): $169-75$.

16. Tavares EL, Anjos LA. Perfil antropométrico da população idosa brasileira. Resultados da Pesquisa Nacional sobre Saúde e Nutrição. Cad Saude Publica 1999; 15(4):327-33.

17. Rauen MS, Moreira EAM, Calvo MCM, Lobo AS. Avaliação do estado nutricional de idosos institucionalizados. Revista de nutric'aPo da PUCCAMP 2008; 21(3):303-10.

18. Mastroeni MF, Erzinger GS, Mastroeni SSBS, Silva NN, Marucci MFN. Perfil demográfico de idosos da cidade de Joinville, Santa Catarina: Estudo de base domiciliar. Revista brasileira de epidemiologia 2007; 10(2):190-201.

19. World Health Organization. Defining the problem of overweight and obesity. In: World 
Health Organization. Obesity: preventing and managing the global epidemic: report of a Who Consultation. Geneva:WHO; 2000. p. 241-3. (WHO Technical Report Series, 894)

20. Lean MEJ, Han TS, Morrison CE. Waist circumference as measure for indicating need for weight management. BMJ 1995; 311:158-61.

21. Callaway WC, Chumlea WC, Bouchard C, Himes JH, Lohman TG, Martin AD, et al. Circumferences. In: Lohman TG, Roche AF, Martorell R, editors. Anthropometric Standardization Reference Manual. Champaign: Human Kinetics; 1988. p. 39-54.

22. Harrison GG, Buskirk ER, Carter JEL, Johnston FE, Lohman TG, Pollock ML et al. Skinfold thicknesses and measurement technique. In: Lohman TG, Roche AF, Martorell R. Anthropometric Standardization Reference Manual. Champaign, Illinois: Human Kinetics Books; 1988. p. 55-68.

23. Resolução 196/96. Conselho Nacional Saúde 1996 outubro 10. Estabelece normas sobre pesquisas envolvendo seres humanos. Diário Oficial da União, [1996 out 10].

24. Lebrão ML. O projeto SABE em São Paulo: uma visão panorâmica. In: Lebrão ML, Duarte YAO, organizadores. SABE: Saúde, Bem-estar e Envelhecimento. O Projeto SABE no Município de São Paulo: uma abordagem inicial. Brasília: Organização Pan-Americana de Saúde; 2003. p. 33-43.
25. Ramos LR, Toniolo JN, Cendoroglo MS, Garcia JT, Najas MS, Perracini M et al. Two-year followup study of elderly residents in S. Paulo, Brazil: methodology and preliminary results. Rev Saude Publica 1998; 32(5):397-407.

26. World Health Organization. Anales da $36^{\circ}$ Reunión del Comitê Asesor de Investigaciones en salud. Encuesta multicentrica: salud, bien estar y envejecimiento (SABE) en América Latina y el Caribe; mayo 2001. Washington (DC): World Health Organization.

27. Marucci MFN, Barbosa AR. Estado nutricional e capacidade física. In: Lebrão ML, Duarte YAO , organizadores. SABE - Saúde, Bem estar e Envelhecimento. O projeto SABE no município de São Paulo: Uma abordagem inicial. Brasília: Organização Pan-Americana da Saúde. 2003. p. 95-117.

28. Groot LCPGM, Sette S, Zajkás G, Carbajal A, Cruz JAA. Nutritional status: anthropometry. Eur J Clin Nutr 1991; 45 (Suppl. 3):31-42.

29. Menezes TM, Marucci MFN. Perfil dos indicadores de gordura e massa muscular corporal dos idosos de Fortaleza, Ceará, Brasil. Cad Saude Publica 2007; 23(12): 2887-95.

30. Santos DM, Sichieri R. Índice de massa corporal e indicadores antropométricos de adiposidade em idosos. Rev Saude Publica 2005; 39(2):163-8.

31. Forster S, Gariballa S. Age as a determinant of nutritional status: a cross sectional study. Nutr J 2005; 4:28-32. 